\title{
A TRANSFORMATION PROPERTY OF THE BOLTZMANN EQUATION*
}

\author{
BY \\ C. -C. WANG \\ Rice University
}

1. The main theorem. In the kinetic theory of gases the governing equation for the phase density function of a simple gas is the Boltzmann equation, which is a nonlinear integrodifferential equation:

$$
\frac{\partial f}{\partial t}+c_{i} \frac{\partial f}{\partial x_{i}}+b_{i} \frac{\partial f}{\partial c_{i}}=\int\|\hat{\mathbf{c}}-\mathbf{c}\|\left(f^{\prime} \hat{f}^{\prime}-f \hat{f}\right) d \hat{w} d \hat{c},
$$

where $t$ denotes the time, $x_{i}, i=1,2,3$, denote the spatial coordinates, and $c_{i}, i=1,2,3$, denote the components of velocity. The usual summation convention is used, so that the repeated index $i$ in (1.1) is summed from 1 to 3 . The notation $f=f\left(t, x_{i}, c_{i}\right)$ is the phase density function whose value represents the number density of the gas molecules on the phase space at the time $t$. Specifically, if $D$ is any domain in the phase space, then the expected number of gas molecules with their phase coordinates bəonging to $D$ at the time $t$ is given by the integral

$$
\int_{\mathbb{D}} f\left(t, x_{i}, c_{i}\right) d x d c
$$

The coefficients $b_{i}, i=1,2,3$, on the left-hand side of (1.1) are the components of the external force field which is acting on the gas at the time $t$.

The integral on the right-hand side of (1.1) is known as the collision integral. Its integrand gives the rate of change of the phase density function due to possible collisions (or encounters) of the molecules with phase coordinates $\left(x_{i}, c_{i}\right)$ and $\left(x_{i}, \hat{c}_{i}\right)$ at time $t$. After the collision the phase coordinates become $\left(x_{i}, c_{i}{ }^{\prime}\right)$ and $\left(x_{i}, \hat{c}_{i}{ }^{\prime}\right)$, respectively, and the symbols $f, \hat{f}, f^{\prime}, \hat{f}^{\prime}$ denote simply the values of $f$ at $\left(t, x_{i}, c_{i}\right),\left(t, x_{i}, \hat{c}_{i}\right),\left(t, x_{i}, c_{i}{ }^{\prime}\right)$, $\left(t, x_{i}, \hat{c}_{i}{ }^{\prime}\right)$, respectively. In order to evaluate the collision integral, we must know the law of intermolecular forces in an encounter. Then by use of analytical mechanics we can determine $\left(c_{i}{ }^{\prime}, \hat{c}_{i}{ }^{\prime}\right)$ as a function of $\left(c_{i}, \hat{c}_{i}\right)$ and the relative position of the molecules before the encounter (cf. [2]). Thus the integral is taken with respect to $\|\hat{\mathbf{c}}-\mathbf{c}\| d \hat{w}$ over all possible relative positions and with respect to $d \hat{c}$ over all possible velocities $\hat{c}_{i}$. The value of the collision integral is then a function of $\left(t, x_{i}, c_{i}\right)$. We shall review the mechanics of an isolated encounter in the next section.

The main purpose of this note is to prove a transformation property of the Boltzmann equation. We define a Euclidean transformation by

\footnotetext{
* Received August 16, 1974. The author is grateful to C. A. Truesdell, who called his attention to this research problem and to reference [1]. The research reported here was supported by a grant of the National Science Foundation to Rice University.
} 


$$
t^{*}=t+\alpha, \quad x_{i}{ }^{*}=Q_{i j}(t) x_{i}+d_{i}(t), \quad c_{i}^{*}=Q_{i j}(t) c_{j}+\dot{Q}_{i j}(t) x_{i}+\dot{d}_{i}(t),
$$

where $\alpha$ is a given real number, $Q_{i j}(t), i, j=1,2,3$, are the components of a given rotation matrix, and $d_{i}(t), i=1,2,3$ are the components of a given translation vector at each time $t$. Both $Q_{i j}(t)$ and $d_{i}(t)$ are assumed to depend smoothly on $t$. We denote the Euclidean transformation by ${ }^{*}$. It is an automorphism of $R^{7}$ such that

$$
*\left(t, x_{i}, c_{i}\right)=\left(t^{*}, x_{i}{ }^{*}, c_{i}{ }^{*}\right) .
$$

Under the Euclidean transformation the phase density function $f\left(t, x_{i}, c_{i}\right)$ is transformed into a new function $f^{*} \equiv *[f]=f \circ *^{-1}$, i.e.

$$
f^{*}\left(t^{*}, x_{i}{ }^{*}, c_{i}{ }^{*}\right)=f\left(t, x_{i}, c_{i}\right),
$$

provided that the arguments are related by (1.3). We call $f^{*}$ the Euclidean transformation of $f$. Our main result is the following

Theorem. If $f\left(t, x_{i}, c_{i}\right)$ is a solution of the Boltzmann equation associated with the external force $b_{i}$, then its Euclidean transformation $f^{*}\left(t^{*}, x_{i}{ }^{*}, c_{i}{ }^{*}\right)$ is a solution of the Boltzmann equation associated with the external force $b_{i}{ }^{*}$ which is related to $b_{i}$ by

$$
b_{i}^{*}=Q_{i j}(t) b_{i}+2 \dot{Q}_{i j}(t) c_{i}+\ddot{Q}_{i j}(t) x_{i}+\ddot{d}_{i}(t),
$$

where the arguments $\left(t^{*}, x_{i}{ }^{*}, c_{i}{ }^{*}\right)$ and $\left(t, x_{i}, c_{i}\right)$ of $b_{i}{ }^{*}$ and $b_{i}$, respectively, are related by (1.3). Thus (1.1) is equivalent to

$$
\frac{\partial f^{*}}{\partial t^{*}}+c_{i}{ }^{*} \frac{\partial f^{*}}{\partial x_{i}{ }^{*}}+b_{i}{ }^{*} \frac{\partial f^{*}}{\partial c_{i}{ }^{*}}=\int\left\|\hat{\mathbf{c}}^{*}-\mathbf{c}^{*}\right\|\left(f^{* \prime} \hat{f}^{* \prime}-f^{*} \hat{f}^{*}\right) d \hat{w}^{*} d \hat{c}^{*} .
$$

We prove the preceding theorem as follows.

2. The collision integral. As explained in the preceding section, the collision integral is an operator on the phase density function $f\left(t, x_{i}, c_{i}\right)$, and the result is also a function of $\left(t, x_{i}, c_{i}\right)$. For definiteness we denote this operator by $\mathrm{C}$, i.e.,

$$
\mathrm{C}[f] \equiv \int\|\hat{\mathbf{c}}-\mathbf{c}\|\left(\hat{f}^{\prime} f^{\prime}-f \hat{f}\right) d \hat{w} d \hat{c},
$$

where a particular but unspecified law of intermolecular forces is understood. We have the following

Lemma 1. The operator C commutes with the operator *:

$$
\mathrm{C} \circ *=* \circ \mathrm{C}
$$

or, equivalently,

$$
\int\left\|\hat{\mathbf{c}}^{*}-\mathbf{c}^{*}\right\|\left(f^{* \prime} \hat{f}^{* \prime}-f^{*} \hat{f}^{*}\right) d \hat{w}^{*} d \hat{c}^{*}=\int\|\hat{\mathbf{c}}-\mathbf{c}\|\left(f^{\prime} \hat{f}^{\prime}-f \hat{f}\right) d \hat{w} d \hat{c}
$$

provided that the arguments $\left(t^{*}, x_{i}{ }^{*}, c_{i}{ }^{*}\right)$ and $\left(t, x_{i}, c_{i}\right)$ are related by (1.3).

To prove this lemma we review first the mechanics of an isolated collision or an isolated encounter of two molecules, which are regarded as two Newtonian mass points. As usual, we assume that the law of intermolecular forces has the component form

$$
h(\|\hat{\mathbf{r}}-\mathbf{r}\|)\left(\hat{r}_{i}-r_{i}\right), \quad i=1,2,3,
$$


where $\hat{\mathbf{r}}$ and $\mathbf{r}$ are the position vectors of the two mass points, and where $h$ is a certain function of the distance $\|\hat{\mathbf{r}}-\mathbf{r}\|$ between the two mass points. The particular form of $h$ characterizes the nature of the interaction, but the proof of the lemma is independent of the choice of $h$.

Suppose that the molecules have asymptotic velocities $\mathbf{c}$ and $\hat{\mathbf{c}}$ before the encounter (i.e., $\mathbf{c}$ and $\hat{\mathbf{c}}$ are the limiting velocities of the molecular trajectories as time tends to negative infinity). Then under the law of forces (2.4) the molecules approach asymptotic velocities $\mathbf{c}^{\prime}$ and $\hat{\mathbf{c}}^{\prime}$ after the encounter (i.e., $\mathbf{c}^{\prime}$ and $\hat{\mathbf{c}}^{\prime}$ are the limiting velocities of the molecular trajectories as time tends to positive infinity). By use of analytical mechanics, we can express $c_{i}{ }^{\prime}$ and $\hat{c}_{i}{ }^{\prime}$ as functions of $c_{i}, \hat{c}_{i}$, and a set of collision parameters, which characterize the relative positions of the molecules before the encounter. For definiteness, let us denote the functional relation between the asymptotic velocities $\left(c_{i}, \hat{c}_{i}\right)$ and $\left(c_{i}{ }^{\prime}, \hat{c}_{i}{ }^{\prime}\right)$ before and after the encounter by

$$
\left(c_{i}{ }^{\prime}, \hat{c}_{i}{ }^{\prime}\right)=\Phi\left(c_{i}, \hat{c}_{i}, \hat{w}\right),
$$

where $\hat{w}$ denotes collectively the collision parameters.

Since the intermolecular forces depend only on the relative position vector $\hat{\mathbf{r}}-\mathbf{r}$ between the molecules, it follows from analytical mechanics that when the asymptotic velocities $\left(c_{i}, \hat{c}_{i}\right)$ before the encounter are changed to $\left(c_{i}^{*}, \hat{c}_{i}{ }^{*}\right)$ with

$$
c_{i}{ }^{*}=Q_{i j} c_{i}+z_{i}, \quad \hat{c}_{i}{ }^{*}=Q_{i i} \hat{c}_{i}+z_{i},
$$

where $Q_{i j}$ and $z_{i}$ are the components of a constant rotation matrix and a constant translational velocity vector, the corresponding asymptotic velocities $\left(c_{i}{ }^{\prime}, \hat{c}_{i}{ }^{\prime}\right)$ after the encounter are transformed to

$$
c_{i}{ }^{* \prime}=Q_{i j} c_{i}{ }^{\prime}+z_{i}, \quad \hat{c}_{i}{ }^{* \prime}=Q_{i j} \hat{c}_{i}{ }^{\prime}+z_{i},
$$

provided that the collision parameters are "rotated" and "translated" by the same $Q_{i j}$ and $z_{i}$ to a new set $\hat{w}^{*}$. It should be noted that, while the asymptotic velocities are the limiting values of the molecular trajectory velocities, which depend on time, the rotation matrix $Q_{i j}$ and the translational veolcity vector $z_{i}$ in (2.6) and (2.7) are not the limiting values of certain time-dependent rotations and translations. Both $Q_{i j}$ and $z_{i}$ are just a constant rotation and a constant translational velocity. The proof of (2.7) is therefore a trivial consequence of analytical mechanics, since the law of forces (2.4) allows the whole trajectories of the molecules be transformed by a constant rotation $Q_{i i}$ and a constant translational velocity $z_{i}$ without violating Newton's equations of motion. In particular, the asymptotic velocities of the molecular trajectories are transformed by (2.6) and (2.7), and the collision parameters are also "rotated" and "translated" accordingly.

As a result of (2.6) and (2.7), we see that the function $\Phi$ in (2.5) for an isolated encounter possesses the following transformation property:

$$
\left(Q_{i j} c_{i}{ }^{\prime}+z_{i}, Q_{i j} \hat{c}_{i}{ }^{\prime}+z_{i}\right)=\Phi\left(Q_{i j} c_{i}+z_{i}, Q_{i j} \hat{c}_{i}+z_{i}, \hat{w}^{*}\right)
$$

for all constant rotation matrices $Q_{i j}$ and all constant translational velocity vectors $z_{i}$. This transformation property of $\Phi$ is the key to the proof of the lemma, as we shall now see.

Since the collision integral (2.1) is taken at a fixed time $t$ and at a fixed position $x_{\imath}$, when we change its argument $\left(t, x_{i}, c_{i}\right)$ to $\left(t^{*}, x_{i}{ }^{*}, c_{i}{ }^{*}\right)$ by (1.3), we can change its inte- 
gration variables $\hat{w}$ and $\hat{c}_{i}$ to $\hat{w}^{*}$ and $\hat{c}_{i}{ }^{*}$, where $\hat{w}^{*}$ is "rotated" and "translated" from $\hat{w}$ by the particular rotation matrix $Q_{i j}$ and the particular translational velocity $z_{i}$ :

$$
Q_{i i}=Q_{i j}(t), \quad z_{i}=\dot{Q}_{i j}(t) x_{i}+\dot{d}_{i}(t) \quad\left(t \text { and } x_{i} \text { are held fixed }\right),
$$

and where $\hat{c}_{i}{ }^{*}$ is likewise transformed from $\hat{c}_{i}$ by

$$
\hat{c}_{i}^{*}=Q_{i j} \hat{c}_{i}+z_{i}=Q_{i j}(t) \hat{c}_{i}+\dot{Q}_{i j}(t) x_{j}+\dot{d}_{i}(t)
$$

Always.remember that both $t$ and $x_{i}$ are held fixed in the collision integral, so that the particular rotation $Q_{i j}=Q_{i j}(t)$ and the particular translational velocity vector $z_{i}=$ $\dot{Q}_{i i}(t) x_{i}+\dot{d}_{i}(t)$ used in the change of variables are both constant. We are only trying to calculate the collision integral by using another set of integration variables.

Now since the change of integration variables (2.10) from $\hat{c}_{i}$ to $\hat{c}_{i}{ }^{*}$ is isochoric on the velocity space, viz.

$$
\frac{\partial\left(\hat{c}_{1}^{*}, \hat{c}_{2}^{*}, \hat{c}_{3}^{*}\right)}{\partial\left(\hat{c}_{1}, \hat{c}_{2}, \hat{c}_{3}\right)}=1
$$

(the same can be said about the transformation of the collision parameters from $\hat{w}$ to $\hat{w}^{*}$ since a "rotation" and a "translation" are volume-preserving on the space of collision parameters), and since the domain of integration is the whole velocity space and the space of all possible collision parameters, by Leibnitz's rule the numerical value of the collision integral is invariant under this transformation. But when we change $\hat{w}$ to $\hat{w}^{*}$ and $\left(c_{i}, \hat{c}_{i}\right)$ to $\left(c_{i}{ }^{*}, \hat{c}_{i}{ }^{*}\right)$, by using the property (2.8) of $\Phi$, we know that the corresponding values of $\left(c_{i}{ }^{\prime}, \hat{c}_{i}{ }^{\prime}\right)$ in the integrand are transformed to $\left(c_{i}{ }^{* \prime}, \hat{c}_{i}{ }^{* \prime}\right)$ in $(2.7)$ with $Q_{i j}$ and $z_{i}$ given by (2.9). As a result, under this change of integration variables the collision integral (2.1) can be rewritten as

$$
\int\left\|\hat{\mathbf{c}}^{*}-\mathbf{c}^{*}\right\|\left(f^{* \prime} \hat{f}^{* \prime}-f^{*} \hat{f}^{*}\right) d \hat{w}^{*} d \hat{c}^{*}
$$

which is precisely the collision integral $C\left[f^{*}\right]$ of the phase density function $f^{*}$. Since the numerical value of the integral is invariant under the change of integration variables, we get (2.3). Thus the lemma is proved.

Remark. For the special case where the molecules are modeled as rigid-elastic spheres of diameter $\sigma$, the collision integral can be written explicitly as

$$
\begin{array}{r}
\frac{1}{2} \sigma^{2} \int\left|\left(\hat{c}_{i}-c_{i}\right) \hat{\alpha}_{i}\right|\left(f\left(t, x_{i}, c_{i}+\hat{\alpha}_{i} \hat{\alpha}_{i}\left(\hat{c}_{i}-c_{i}\right)\right) f\left(t, x_{i}, \hat{c}_{i}-\hat{\alpha}_{i} \hat{\alpha}_{i}\left(\hat{c}_{i}-c_{i}\right)\right)\right. \\
\left.-f\left(t, x_{i}, c_{i}\right) f\left(t, x_{i}, \hat{c}_{i}\right)\right) d \hat{w} d \hat{c},
\end{array}
$$

where $\hat{\alpha}_{i}$ denotes a unit vector and $d \hat{w}$ denotes the differential element of solid angle on the unit sphere at $\hat{\alpha}_{i}$. The change of variables from $\left(t, x_{i}, c_{i}\right)$ to $\left(t^{*}, x_{i}{ }^{*}, c_{i}{ }^{*}\right)$ by (1.3), the change of integration variables from $\hat{c}_{i}$ to $\hat{c}_{i}{ }^{*}$ by $(2.10)$, from $\hat{\alpha}_{i}$ to $\hat{\alpha}_{i}{ }^{*}$ by

$$
\hat{\alpha}_{i}^{*}=Q_{i j} \hat{\alpha}_{j} \equiv Q_{i j}(t) \hat{\alpha}_{i}
$$

and from the element of solid angle $d \hat{w} \hat{\alpha}_{i}$ to $d \hat{w}^{*} \hat{\alpha}_{i}{ }^{*}$ jointly reduce the integral (2.13) to the integral 


$$
\begin{aligned}
\frac{1}{2} \sigma^{2} \int\left|\left(\hat{c}_{i}{ }^{*}-c_{i}{ }^{*}\right) \hat{\alpha}_{i}{ }^{*}\right|\left(f ^ { * } \left(t^{*}, x_{i}{ }^{*}, c_{i}{ }^{*}\right.\right. & \left.+\hat{\alpha}_{i}{ }^{*} \hat{\alpha}_{i}{ }^{*}\left(\hat{c}_{i}{ }^{*}-c_{i}{ }^{*}\right)\right) \\
& \cdot f^{*}\left(t^{*}, x_{i}{ }^{*}, \hat{c}_{i}{ }^{*}-\hat{\alpha}_{i}{ }^{*} \alpha_{i}{ }^{*}\left(\hat{c}_{i}{ }^{*}-c_{i}{ }^{*}\right)\right) \\
& \left.-f^{*}\left(t^{*}, x_{i}{ }^{*}, c_{i}{ }^{*}\right) f^{*}\left(t^{*}, x_{i}{ }^{*}, \hat{c}_{i}{ }^{*}\right)\right) d \hat{w}^{*} d \hat{c}^{*},
\end{aligned}
$$

which is precisely the collision integral of the Euclidean transformation $f^{*}$ of $f$. Notice that the key to the form (2.15) is the fact that

$$
c_{i}^{*}+\hat{\alpha}_{i}^{*} \hat{\alpha}_{i}^{*}\left(\hat{c}_{i}^{*}-c_{i}^{*}\right)=Q_{i j}(t)\left(c_{i}+\hat{\alpha}_{i} \hat{\alpha}_{k}\left(\hat{c}_{k}-c_{k}\right)+\dot{Q}_{i j}(t) x_{i}+\dot{d}_{i}(t)\right.
$$

and

$\hat{c}_{i}{ }^{*}-\hat{\alpha}_{i}{ }^{*} \hat{\alpha}_{i}^{*}\left(\hat{c}_{i}{ }^{*}-c_{i}^{*}\right)=Q_{i j}(t)\left(\hat{c}_{i}-\hat{\alpha}_{j} \hat{\alpha}_{k}\left(\hat{c}_{k}-c_{k}\right)\right)+\dot{Q}_{i j}(t) x_{i}+d_{i}(t)$

which imply, respectively, that

$$
\left(t^{*}, x_{i}{ }^{*}, c_{i}^{*}+\hat{\alpha}_{i}^{*} \hat{\alpha}_{i}^{*}\left(\hat{c}_{i}^{*}-c_{i}^{*}\right)\right)=*\left(t, x_{i}, \hat{\alpha}_{i} \hat{\alpha}_{i}\left(\hat{c}_{i}-c_{i}\right)\right)
$$

and

$$
\left(t^{*}, x_{i}{ }^{*}, \hat{c}_{i}{ }^{*}-\hat{\alpha}_{i}^{*} \hat{\alpha}_{i}^{*}\left(\hat{c}_{i}^{*}-c_{i}^{*}\right)\right)=*\left(t, x_{i}, \hat{\alpha}_{i} \hat{\alpha}_{i}\left(\hat{c}_{i}-c_{i}\right)\right) .
$$

The condition (2.16) is the special case of the general condition (2.8) for collisions of rigid-elastic spheres.

Before closing this section we should point out that while the transformation property (2.8) of the collision relation $\Phi$ is valid for a constant rotation $Q_{i j}$ and a constant translational velocity $z_{i}$ only, its consequence: the transformation property (2.3) of the collision integral, is valid for any Euclidean transformation (1.3), where $Q_{i j}(t)$ and $d_{i}(t)$ are not constant but arbitrary smooth functions of $t$. The use of the asymptotic velocities in the collision integral is one of several basic assumptions admitted in the derivation of the Boltzmann equation. Clearly this assumption cannot possibly be exact except in the rigid-elastic sphere case where the collision is an impact, since on the one hand it generally takes an infinitely long period of time and an infinitely long curve in space to achieve the asymptotic velocities, but on the other hand both $t$ and $x_{i}$ are held fixed in the collision integral. However, the discrepancy that exists between the mathematical definition of the collision integral and the actual physical nature of molecular collisions in a gas is not of concern to us here. What we have shown is simply a mathematical property of the collision integral-as it is defined mathematically in the kinetic theory.

The logical inconsistency in the definition of the collision integral amounts to the use of the collision relation $\Phi$ as if it were an impact relation. Equivalently, there are two separate sets of space-time variables involved, one set for the derivation of the collision relation $\Phi$ and one set for the collision integral C. Once the collision relation $\Phi$ is derived, the first set of space-time variables is completely forgotten. While the rotation $Q_{i j}$ and the translational velocity $z_{i}$ must be constant with respect to the first set of space-time variables, they are not necessarily constant with respect to the second set of space-time variables. Indeed, once $\Phi$ is derived, the condition (2.8) is a mathematical identity for $\Phi$ and is valid for all $\left[Q_{i i}\right] \in S \mathcal{S}(3)$ and all $\left(z_{i}\right) \in \mathcal{R}^{3}$. In particular, we can apply (2.8) to $\left[Q_{i j}(t)\right]$ and $\left[z_{i}\left(t, x_{i}\right)\right)$, where $x_{i}$ and $t$ are the space-time variables in the second set.

3. The streaming terms. The left-hand side of the Boltzmann equation (1.1) is known as the streaming terms. It defines a differential operator on the phase density 
function $f\left(t, x_{i}, c_{i}\right)$, and the result is a function of $\left(t, x_{i}, c_{i}\right)$. For definiteness, we denote this operator by $D$, i.e.

$$
\mathrm{D}[f]=\frac{\partial f}{\partial t}+c_{i} \frac{\partial f}{\partial x_{i}}+b_{i} \frac{\partial f}{\partial c_{i}},
$$

where a particular external force field $b_{i}$ is understood. For obvious reason we denote the streaming terms on the left-hand side of $(1.7)$ by $D^{*}\left[f^{*}\right]$, i.e.,

$$
D^{*}\left[f^{*}\right]=\frac{\partial f^{*}}{\partial t^{*}}+c_{i}{ }^{*} \frac{\partial f^{*}}{\partial x_{i}{ }^{*}}+b_{i}{ }^{*} \frac{\partial f^{*}}{\partial c_{i}{ }^{*}},
$$

where the external force field $b_{i}^{*}$ is given by (1.6). Of course, $D^{*}\left[f^{*}\right]$ is a function of $\left(t^{*}, x_{i}{ }^{*}, c_{i}{ }^{*}\right)$. We have the following

Lemma 2. $D^{*}\left[f^{*}\right]$ is the Euclidean transformation of $D[f]$, i.e.,

$$
D^{*} \circ *=* \circ D
$$

or, equivalently,

$$
\frac{\partial f^{*}}{\partial t^{*}}+c_{i}{ }^{*} \frac{\partial f^{*}}{\partial x_{i}{ }^{*}}+b_{i}{ }^{*} \frac{\partial f^{*}}{\partial c_{i}{ }^{*}}=\frac{\partial f}{\partial t}+c_{i} \frac{\partial f}{\partial x_{i}}+b_{i} \frac{\partial f}{\partial c_{i}}
$$

provided that $\left(t^{*}, x_{i}{ }^{*}, c_{i}{ }^{*}\right)$ and $\left(t, x_{i}, c_{i}\right)$ are related by (1.3).

To prove this lemma we differentiate (1.5) with respect to $t, x_{i}$, and $c_{i}$, respectively, and we use the chain rule based on (1.3):

$$
\begin{aligned}
& \frac{\partial f}{\partial t}=\frac{\partial f^{*}}{\partial t^{*}}+\left(\dot{Q}_{i j} x_{i}+\dot{d}_{i}\right) \frac{\partial f^{*}}{\partial x_{i}{ }^{*}}+\left(\dot{Q}_{i j} c_{i}+\ddot{Q}_{i j} x_{i}+\ddot{d}_{i}\right) \frac{\partial f^{*}}{\partial c_{i}{ }^{*}}, \\
& \frac{\partial f}{\partial x_{i}}=Q_{i i} \frac{\partial f^{*}}{\partial x_{i}{ }^{*}}+\dot{Q}_{i i} \frac{\partial f^{*}}{\partial c_{i}{ }^{*}}, \\
& \frac{\partial f}{\partial c_{i}}=Q_{i i} \frac{\partial f^{*}}{\partial c_{i}{ }^{*}} .
\end{aligned}
$$

Now, forming the streaming terms $D[f]$ and using the formula (1.6) for the external force field $b_{i}{ }^{*}$, we obtain (3.4). Thus the lemma is proved.

The main theorem now follows directly from Lemmas 1 and 2 , since numerically the two sides of (1.7) are equal to the corresponding two sides of (1.1) provided that the arguments $\left(t^{*}, x_{i}{ }^{*}, c_{i}{ }^{*}\right)$ of the former are related to the arguments $\left(t, x_{i}, c_{i}\right)$ of the latter by (1.3).

Remark. In the physical interpretation our main theorem says simply that if a given process of a gas, as characterized by the phase density function $f\left(t, x_{i}, c_{i}\right)$, is compatible with the external force field $b_{i}$ according to the Boltzmann equation, then the process, which is the result of superimposing a rigid motion on the given process as characterized by the Euclidean transformation $f^{*}\left(t^{*}, x_{i}{ }^{*}, c_{i}{ }^{*}\right)$, is compatible with the external force field $b_{i}{ }^{*}$, which is related to $b_{i}$ by (1.6). This result is only natural, since (1.6) is also the transformation law for the accelerations of the molecular trajectories associated with the Euclidean transformation (1.3), i.e.,

$$
a_{i}{ }^{*}=Q_{i i}(t) a_{i}+2 \dot{Q}_{i j}(t) c_{i}+\ddot{Q}_{i j}(t) x_{i}+\ddot{d}_{i}(t), \quad i=1,2,3 .
$$

By Newton's law of motion $a_{i}$ is equal to $b_{i}$ and $a_{i}{ }^{*}$ is equal to $b_{i}{ }^{*}$. As a result, if a given 
molecular trajectory is compatible with $b_{i}$, then its Euclidean transformation is compatible with $b_{i}{ }^{*}$. In this sense our main theorem is consistent with the transformation property of Newton's equations of motion.

It should be noted, however, that the main purpose of proving the transformation property is not to find any new exact solution of the Boltzmann equation. True, our main theorem says that the Euclidean transformation of any exact solution of the Boltzmann equation relative to an external force $\mathbf{b}$ is an exact solution of the Boltzmann equation relative to a new external force $b^{*}$, which is related to $b$ by (1.6). But the point is, when $\mathbf{b}$ has a simple form such $\mathbf{a s} \mathbf{b}=\mathbf{0}, \mathbf{b}^{*}$ is generally rather complicated. The only useful case is probably the case when $Q_{i j}(t)=\delta_{i j}$ and $\ddot{d}_{i}(t)=g_{i}=$ constant. In this case, if $b_{i}=0$, then $b_{i}{ }^{*}=g_{i}$. So any exact solution of the Boltzmann equation under no external force can be transformed by a special Euclidean transformation:

$$
t^{*}=t+\alpha, \quad x_{i}^{*}=x_{i}+\frac{1}{2} g_{i} t^{2}+h_{i} t+l_{i}, \quad c_{i}^{*}=c_{i}+g_{i} t+h_{i}
$$

into an exact solution of the Boltzmann equation under a constant external force $b_{i}{ }^{*}=g_{i}$. Other than this simple case, the new external force $b_{i}{ }^{*}$ required to maintain the new solution $f^{*}$ cannot really be produced physically in a laboratory.

The main application of the transformation property is to prove that (1.7) is the governing equation of the phase density function relative to an arbitrary frame of reference which is related to an inertial frame by the Euclidean transformation (1.3). Under such a change of frame the new external force $b_{i}{ }^{*}$ given by (1.6) is just the apparent external force of the original external force $b_{i}$ in the new frame. In this sense the equation (1.7) is stated without proof by Müller [1, Eq. (2.1)], who used it to derive the Maxwellian iterations of the macroscopic quantities relative to an arbitrary frame.

If we use Maxwell's definitions for macroscopic quantities such as the average velocity, the average acceleration, the stress tensor, the heat flux vector, the temperature, the internal energy density, etc. in any given process of a gas, then the equivalent sets of Maxwell's equations of transport obtained from (1.1) and (1.7), respectively, are consistent with a basic consequence of the principle of objectivity in continuum mechanics. We shall explore the relation between our main theorem here and the principle of objectivity in continuum mechanics in another paper, where the transformation properties of the approximate solutions of the Boltzmann equation, obtained by various iterative processes, will be considered.

\section{REFERENCES}

[1] I. Müller, Arch. Rational Mech. Anal. 45, 241-250 (1972)

[2] J. H. Ferziger and H. G. Kaper, Mathematical theory of transport processes in gases, North-Holland Publishing Co. (1972) 